\title{
Evaluation of Reliability and Availability Characteristics of a Repairable System with Active Parallel Units
}

\author{
Ibrahim Yusuf $^{*}$, Fatima Salman Koki ${ }^{2}$ \\ ${ }^{1}$ Department of Mathematical Sciences, Bayero University, Kano, Nigeria \\ ${ }^{2}$ Department of Physics, Bayero University, Kano \\ Email: *Ibrahimyusuffagge@gmail.com, FatimaSK2775@gmail.com \\ Received June 5, 2013; revised July 15, 2012; accepted July 22, 2013
}

Copyright (C) 2013 Ibrahim Yusuf, Fatima Salman Koki. This is an open access article distributed under the Creative Commons Attribution License, which permits unrestricted use, distribution, and reproduction in any medium, provided the original work is properly cited.

\begin{abstract}
In this paper, we study the reliability and availability characteristics of a repairable system consisting of two subsystems $A$ and $B$ in series. Subsystem A consists of two units $A_{1}$ and $A_{2}$ operating in active parallel while subsystem $B$ is a single unit. Failure and repair times are assumed exponential. The explicit expressions of reliability and availability characteristics like mean time to system failure (MTSF), system availability, busy period and profit function are derived using Kolmogorov forward equations method. Various cases are analyzed graphically to investigate the impacts of system parameters on MTSF, availability, busy period and profit function.
\end{abstract}

Keywords: Active Parallel; Reliability; Availability; Mean Time to System Failure

\section{Introduction}

Reliability is vital for proper utilization and maintenance of any system. It involves techniques for increasing system effectiveness through reducing failure frequency and maintenance-cost minimization. Adequate maintenance management is vital in reducing the adverse effect of equipment failures and maintenance cost and in maximizing equipment availability. The increase in equipment availability means less maintenance cost, higher productivity and higher profit. There are systems of three units in which two/three units are sufficient to perform the entire function of the system. Such systems are called 2out-of-3 or 3-out-of-3 redundant systems. These systems have wide application in the real world. The communication system with three transmitters can be sited as a good example of 2-out-of-3 redundant system. One of the commonly used forms of redundancy is active parallel system, which often finds applications in various industrial or other types of setup. Due to their importance in industries and system design, models of redundant systems as well as methods of evaluating system reliability and availability have been researched in order to improve the system effectiveness (see, for instance, [1] and [2]). S. V. Amari et al. [3] show that the reliability of systems

\footnotetext{
${ }^{*}$ Corresponding author.
}

subject to imperfect fault-coverage decreases after a certain level of active redundancy. K.-H. Wang and B. D. Sivazlian [4] deal with the reliability characteristics of a multiple-server unit system with warm standby units with exponential failure and exponential repair time distributions. Steady-state availability and the mean time to system failure of a repairable system with warm standbys plus balking and reneging were studied by J.-C. Ke and K.-H. Wang [5,6]. K.-H. Wang et al. [7] deals with the reliability and sensitivity analysis of a system with $\mathrm{M}$ operating machines, $\mathrm{S}$ warm standbys, and a repairable service station. The problem considered in this paper is different from the work of K. M. El-Said et al. [1,2]. The main contribution of this paper is two-fold. The first is to develop the explicit expressions for MTSF, system availability, busy period and profit function. The second is to perform a parametric investigation of various system parameters on MTSF, system availability and profit function and capture their effects on MTSF, availability, busy period and profit function. The rest of the paper is organized as follows. Section 2 gives the notations, assumptions of the study, the reliability block diagram and the states of the system. Section 3 gives the states of the system. Section 4 deals with models formulation. The results of our numerical simulations are presented and discussed in Section 5. Section 6 is the conclusion of the paper. 


\section{Notations and Assumptions}

\subsection{Notations}

$a i$ : Type $i$ repair rate of unit $A i$ in operation, $i=1,2$. $\beta i$ : Type $i$ failure rate of unit in operation $A i, i=1,2$. $\eta$ : Type III repair rate of subsystem $B$ in operation. $\delta$ : Type III failure rate of subsystem $B$ in operation.

\subsection{Assumptions}

1) The systems consist of two dissimilar subsystems $A$ and $B$ in series; 2) Subsystem $A$ consist of two units $A 1$ and $A 2$ in active parallel; 3) The system work in a re-duced capacity at the failure of unit $A 1$ or $A 2$; 4) Subsys-tem $B$ is a single unit; 5) The systems have two states: normal and failure. 6) Unit failure and repair rates are constant; 7) Repair is as good as new; 8) Failure and re-pair time are assumed exponential; 9) The system fail at the failure of $A 1$ and $A 2$ or subsystem $B ; 10$ ) The system is under the attention of one repairman.

\section{States of the System}

1) State $S 0$ : Units $A 1, A 2$ and subsystem $B$ are working, the system is working. 2) State $S 1$ : Unit $A 1$ is under Type I repair, unit $A 2$ is working, subsystem $B$ is working, and the system is working. 3) State $S 2$ : Unit $A 1$ is working, unit $A 2$ is under Type II repair, subsystem $B$ is working, and the system is working. 4) State $S 3$ : Unit $A 1$ and $A 2$ are good, subsystem $B$ is under Type III repair, and the system failed. 5) State $S 4$ : Unit $A 1$ is under Type I repair, unit $A 2$ is good, subsystem $B$ is under Type III repair, and the system failed. 6) State $S 5$ : Unit $A 1$ is under Type I repair, unit $A 2$ is under Type I repair, subsystem $B$ is good, and the system failed. 7) State $S 6$ : Unit $A 1$ is under Type I repair, unit $A 2$ is under Type II, subsystem $B$ is good, and the system failed. 8) State $S 7$ : Unit $A 1$ is good, unit $A 2$ is under Type II repair, subsystem $B$ is under Type III repair, and the system failed. 9) State S8: Unit $A 1$ is under Type II repair, unit $A 2$ is under Type II, subsystem $B$ is good, and the system failed.

\section{Models Formulation}

\subsection{Mean Time to System Failure for System}

Let $P(t)$ be the probability row vector at time $t$, then the initial conditions for this problem are as follows:

$$
\begin{aligned}
P(0) & =\left[\begin{array}{l}
P_{0}(0), P_{1}(0), P_{2}(0), P_{3}(0), P_{4}(0), \\
P_{5}(0), P_{6}(0), P_{7}(0), P_{8}(0)
\end{array}\right] \\
& =[1,0,0,0,0,0,0,0,0]
\end{aligned}
$$

we obtain the following system of differential equations from Figure 1 above:

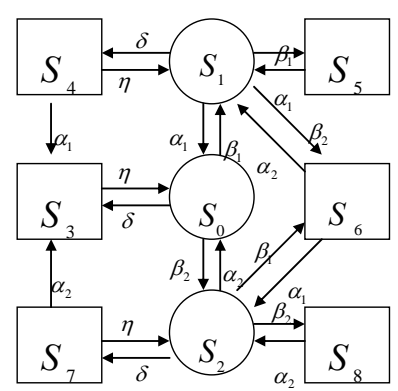

Figure 1. Schematic diagram of the System.

$$
\begin{aligned}
\frac{\mathrm{d} P_{0}(t)}{\mathrm{d} t}= & -\left(\beta_{1}+\beta_{2}+\delta\right) P_{0}(t)+\alpha_{1} P_{1}(t) \\
& +\alpha_{2} P_{2}(t)+\eta P_{3}(t) \\
\frac{\mathrm{d} P_{1}(t)}{\mathrm{d} t}= & -\left(\alpha_{1}+\beta_{1}+\beta_{2}+\delta\right) P_{1}(t)+\beta_{1} P_{0}(t) \\
& +\eta P_{4}(t)+\alpha_{1} P_{5}(t)+\alpha_{2} P_{6}(t) \\
\frac{\mathrm{d} P_{2}(t)}{\mathrm{d} t}= & -\left(\alpha_{2}+\beta_{1}+\beta_{2}+\delta\right) P_{2}(t)+\beta_{2} P_{0}(t) \\
& +\alpha_{1} P_{6}(t)+\eta P_{7}(t)+\alpha_{2} P_{8}(t) \\
\frac{\mathrm{d} P_{3}(t)}{\mathrm{d} t}= & -\eta P_{3}(t)+\delta P_{0}(t)+\alpha_{1} P_{4}(t)+\alpha_{2} P_{7}(t) \\
\frac{\mathrm{d} P_{4}(t)}{\mathrm{d} t}= & -\left(\alpha_{1}+\eta\right) P_{4}(t)+\delta P_{1}(t) \\
\frac{\mathrm{d} P_{5}(t)}{\mathrm{d} t}= & -\alpha_{1} P_{5}(t)+\beta_{1} P_{1}(t) \\
\frac{\mathrm{d} P_{6}(t)}{\mathrm{d} t}= & -\left(\alpha_{1}+\alpha_{2}\right) P_{6}(t)+\beta_{2} P_{1}(t)+\beta_{1} P_{2}(t) \\
\frac{\mathrm{d} P_{7}(t)}{\mathrm{d} t}= & -\left(\alpha_{2}+\eta\right) P_{7}(t)+\delta P_{2}(t) \\
\frac{\mathrm{d} P_{8}(t)}{\mathrm{d} t}= & -\alpha_{2} P_{8}(t)+\beta_{2} P_{2}(t)
\end{aligned}
$$

The above system of differential equations can be written in matrix form as

$$
\dot{P}=A P
$$

where

$$
A=\left[\begin{array}{ccccccccc}
h_{1} & \alpha_{1} & \alpha_{2} & \eta & 0 & 0 & 0 & 0 & 0 \\
\beta_{1} & h_{2} & 0 & 0 & \eta & \alpha_{1} & \alpha_{2} & 0 & 0 \\
\beta_{2} & 0 & h_{3} & 0 & 0 & 0 & \alpha_{1} & \eta & \alpha_{2} \\
\delta & 0 & 0 & -\eta & \alpha_{1} & 0 & 0 & \alpha_{2} & 0 \\
0 & \delta & 0 & 0 & h_{4} & 0 & 0 & 0 & 0 \\
0 & \beta_{1} & 0 & 0 & 0 & -\alpha_{1} & 0 & 0 & 0 \\
0 & \beta_{2} & \beta_{1} & 0 & 0 & 0 & h_{5} & 0 & 0 \\
0 & 0 & \delta & 0 & 0 & 0 & 0 & h_{6} & 0 \\
0 & 0 & \beta_{2} & 0 & 0 & 0 & 0 & 0 & -\alpha_{2}
\end{array}\right]
$$


where

$$
\begin{aligned}
& h_{1}=-\left(\beta_{1}+\beta_{2}+\delta\right) \\
& h_{2}=-\left(\alpha_{1}+\beta_{1}+\beta_{2}+\delta\right) \\
& h_{3}=-\left(\alpha_{2}+\beta_{1}+\beta_{2}+\delta\right) \\
& h_{4}=-\left(\alpha_{1}+\eta\right) \\
& h_{5}=-\left(\alpha_{1}+\alpha_{2}\right) \\
& h_{6}=-\left(\alpha_{2}+\eta\right)
\end{aligned}
$$

It is difficult to evaluate the transient solutions, hence we follow [8,9], the procedure to develop the explicit expression for MTSF is to delete the fourth row to ninth, fourth to ninth column of matrix $A$ and take the transpose to produce a new matrix, say $Q$. The expected time to reach an absorbing state is obtained from

$$
E\left[T_{P(0) \rightarrow P(\text { absorbing })}\right]=P(0)\left(-Q^{-1}\right)\left(\begin{array}{l}
1 \\
1 \\
1
\end{array}\right)=\operatorname{MTSF}=\frac{N_{1}}{D_{1}}
$$

where

$$
\begin{aligned}
& Q=\left[\begin{array}{ccc}
-\left(\beta_{1}+\beta_{2}+\delta\right) & \beta_{1} & \beta_{2} \\
\alpha_{1} & -\left(\alpha_{1}+\beta_{1}+\beta_{2}+\delta\right) & 0 \\
\alpha_{2} & 0 & -\left(\alpha_{2}+\beta_{1}+\beta_{2}+\delta\right)
\end{array}\right] \\
& N_{1}=\left(\alpha_{1}+\beta_{1}+\beta_{2}+\delta\right)\left(\alpha_{2}+\beta_{1}+\beta_{2}+\delta\right) \\
& \quad+\beta_{1}\left(\alpha_{2}+\beta_{1}+\beta_{2}+\delta\right)+\beta_{2}\left(\alpha_{1}+\beta_{1}+\beta_{2}+\delta\right) \\
& D_{1}= \alpha_{1} \alpha_{2} \delta+2 \alpha_{2} \beta_{1} \delta+\alpha_{1} \beta_{1} \delta+\alpha_{2} \beta_{2} \delta+\beta_{1}^{3}+\beta_{2}^{3}+\delta^{3} \\
&+\alpha_{2} \beta_{1}^{2}+3 \beta_{1}^{2} \beta_{2}+3 \beta_{1}^{2} \delta+3 \beta_{1} \beta_{2}^{2}+3 \beta_{1} \delta^{2} \\
&+\alpha_{1} \beta_{2}^{2}+3 \beta_{2}^{2} \delta+3 \beta_{2} \delta^{2}+\alpha_{1} \delta^{2}+\alpha_{2} \delta^{2}+\alpha_{1} \beta_{1} \beta_{2} \\
&+\alpha_{2} \beta_{1} \beta_{2}+6 \beta_{1} \beta_{2} \delta+2 \alpha_{1} \beta_{2} \delta
\end{aligned}
$$

\subsection{Availability Analysis}

For the availability case of Figure 2 following [1,10] using the initial condition in subsection 4.1 for this system.

$$
\begin{aligned}
P(0) & =\left[\begin{array}{l}
P_{0}(0), P_{1}(0), P_{2}(0), P_{3}(0), P_{4}(0), \\
P_{5}(0), P_{6}(0), P_{7}(0), P_{8}(0)
\end{array}\right] \\
& =[1,0,0,0,0,0,0,0,0]
\end{aligned}
$$

The system of differential equations in (1) for the system above can be expressed in matrix form as:

Let $T$ be the time to failure of the system. The steady-state availability is given by

$$
A_{T}=P_{0}(\infty)+P_{1}(\infty)+P_{2}(\infty)
$$

In steady state, the derivatives of state probabilities become zero,

$$
\boldsymbol{A P}(\infty)=\mathbf{0}
$$

$\left[\begin{array}{ccccccccc}h_{1} & \alpha_{1} & \alpha_{2} & \eta & 0 & 0 & 0 & 0 & 0 \\ \beta_{1} & h_{2} & 0 & 0 & \eta & \alpha_{1} & \alpha_{2} & 0 & 0 \\ \beta_{2} & 0 & h_{3} & 0 & 0 & 0 & \alpha_{1} & \eta & \alpha_{2} \\ \delta & 0 & 0 & -\eta & \alpha_{1} & 0 & 0 & \alpha_{2} & 0 \\ 0 & \delta & 0 & 0 & h_{4} & 0 & 0 & 0 & 0 \\ 0 & \beta_{1} & 0 & 0 & 0 & -\alpha_{1} & 0 & 0 & 0 \\ 0 & \beta_{2} & \beta_{1} & 0 & 0 & 0 & h_{5} & 0 & 0 \\ 0 & 0 & \delta & 0 & 0 & 0 & 0 & h_{6} & 0 \\ 0 & 0 & \beta_{2} & 0 & 0 & 0 & 0 & 0 & -\alpha_{2}\end{array}\right]\left[\begin{array}{c}P_{0} \\ P_{1} \\ P_{2} \\ P_{3} \\ P_{4} \\ P_{5} \\ P_{6} \\ P_{7} \\ P_{8}\end{array}\right]=\left[\begin{array}{l}0 \\ 0 \\ 0 \\ 0 \\ 0 \\ 0 \\ 0 \\ 0 \\ 0\end{array}\right]$

using the normalizing condition

$$
P_{0}(\infty)+P_{1}(\infty)+P_{2}(\infty)+P_{3}(\infty)+\cdots+P_{8}(\infty)=1
$$

We substitute (6) in the last of row of (5). The resulting matrix is

$$
\left[\begin{array}{c}
\dot{P}_{0} \\
\dot{P}_{1} \\
\dot{P}_{2} \\
\dot{P}_{3} \\
\dot{P}_{4} \\
\dot{P}_{5} \\
\dot{P}_{6} \\
\dot{P}_{7} \\
\dot{P}_{8}
\end{array}\right]=\left[\begin{array}{ccccccccc}
h_{1} & \alpha_{1} & \alpha_{2} & \eta & 0 & 0 & 0 & 0 & 0 \\
\beta_{1} & h_{2} & 0 & 0 & \eta & \alpha_{1} & \alpha_{2} & 0 & 0 \\
\beta_{2} & 0 & h_{3} & 0 & 0 & 0 & \alpha_{1} & \eta & \alpha_{2} \\
\delta & 0 & 0 & -\eta & \alpha_{1} & 0 & 0 & \alpha_{2} & 0 \\
0 & \delta & 0 & 0 & h_{4} & 0 & 0 & 0 & 0 \\
0 & \beta_{1} & 0 & 0 & 0 & -\alpha_{1} & 0 & 0 & 0 \\
0 & \beta_{2} & \beta_{1} & 0 & 0 & 0 & h_{5} & 0 & 0 \\
0 & 0 & \delta & 0 & 0 & 0 & 0 & h_{6} & 0 \\
0 & 0 & \beta_{2} & 0 & 0 & 0 & 0 & 0 & -\alpha_{2}
\end{array}\right]\left[\begin{array}{c}
P_{0} \\
P_{1} \\
P_{2} \\
P_{3} \\
P_{4} \\
P_{5} \\
P_{6} \\
P_{7} \\
P_{8}
\end{array}\right]
$$




$$
\left[\begin{array}{ccccccccc}
h_{1} & \alpha_{1} & \alpha_{2} & \eta & 0 & 0 & 0 & 0 & 0 \\
\beta_{1} & h_{2} & 0 & 0 & \eta & \alpha_{1} & \alpha_{2} & 0 & 0 \\
\beta_{2} & 0 & h_{3} & 0 & 0 & 0 & \alpha_{1} & \eta & \alpha_{2} \\
\delta & 0 & 0 & -\eta & \alpha_{1} & 0 & 0 & \alpha_{2} & 0 \\
0 & \delta & 0 & 0 & h_{4} & 0 & 0 & 0 & 0 \\
0 & \beta_{1} & 0 & 0 & 0 & -\alpha_{1} & 0 & 0 & 0 \\
0 & \beta_{2} & \beta_{1} & 0 & 0 & 0 & h_{5} & 0 & 0 \\
0 & 0 & \delta & 0 & 0 & 0 & 0 & h_{6} & 0 \\
1 & 1 & 1 & 1 & 1 & 1 & 1 & 1 & 1
\end{array}\right]\left[\begin{array}{c}
P_{0}(\infty) \\
P_{1}(\infty) \\
P_{2}(\infty) \\
P_{3}(\infty) \\
P_{4}(\infty) \\
P_{5}(\infty) \\
P_{6}(\infty) \\
P_{7}(\infty) \\
P_{8}(\infty)
\end{array}\right]=\left[\begin{array}{l}
0 \\
0 \\
0 \\
0 \\
0 \\
0 \\
0 \\
0 \\
1
\end{array}\right]
$$

Thus, the expression for $A_{T}$ is

$$
A_{T}=\frac{N_{2}}{D_{2}}
$$

where

$$
\begin{aligned}
& N_{2}=\alpha_{1}^{2} \alpha_{2}^{2} \eta\left(\begin{array}{l}
2 \alpha_{1} \alpha_{2} \delta+\alpha_{2} \beta_{1} \delta+\beta_{2} \eta \delta+\alpha_{1} \delta^{2}+\alpha_{2} \delta^{2}+\alpha_{1} \beta_{2} \delta+\beta_{1} \eta \delta+2 \alpha_{1} \eta \delta+\alpha_{2}^{2} \delta+\beta_{2} \eta^{2}+\beta_{1} \eta^{2}+\alpha_{1} \beta_{1} \eta+\alpha_{2} \beta_{2} \eta \\
+2 \alpha_{2} \eta \delta+\alpha_{1} \beta_{2} \eta+\alpha_{2} \beta_{1} \eta+\alpha_{1}^{2} \delta+\alpha_{1} \alpha_{2} \beta_{2}+\alpha_{1} \alpha_{2} \beta_{1}+\alpha_{1} \eta^{2}+\alpha_{1} \alpha_{2}^{2}+\alpha_{1}^{2} \alpha_{2}+\alpha_{2} \eta^{2}+2 \alpha_{1} \alpha_{2} \eta+\alpha_{1}^{2} \eta+\alpha_{2}^{2} \eta
\end{array}\right) \\
& +\alpha_{1} \alpha_{2}^{2} \beta_{1} \eta\left(\begin{array}{l}
\alpha_{1} \eta^{2}+\alpha_{2} \eta^{2}+\beta_{1} \eta^{2}+\beta_{2} \eta^{2}+\alpha_{1}^{2} \eta+2 \alpha_{1} \alpha_{2} \eta+\alpha_{1} \beta_{2} \eta+\alpha_{1} \eta \delta+\alpha_{1} \beta_{1} \eta+\alpha_{2}^{2} \eta \\
+\alpha_{2} \beta_{2} \eta+\alpha_{2} \eta \delta+\alpha_{2} \beta_{1} \eta+\alpha_{1}^{2} \alpha_{2}+\alpha_{1}^{2} \delta+\alpha_{1} \alpha_{2}^{2}+\alpha_{1} \alpha_{2} \beta_{2}+\alpha_{1} \alpha_{2} \delta+\alpha_{1} \alpha_{2} \beta_{1}
\end{array}\right) \\
& +\alpha_{1}^{2} \alpha_{2} \beta_{2} \eta\left(\begin{array}{l}
\alpha_{1} \eta^{2}+\alpha_{2} \eta^{2}+\beta_{2} \eta^{2}+\beta_{1} \eta^{2}+\alpha_{1}^{2} \eta+2 \alpha_{1} \alpha_{2} \eta+\alpha_{1} \eta \delta+\alpha_{1} \beta_{2} \eta+\alpha_{1} \beta_{1} \eta+\alpha_{2}^{2} \eta \\
+\alpha_{2} \eta \delta+\alpha_{2} \beta_{2} \eta+\alpha_{2} \beta_{1} \eta+\alpha_{1}^{2} \alpha_{2}+\alpha_{1} \alpha_{2}^{2}+\alpha_{1} \alpha_{2} \delta+\alpha_{1} \alpha_{2} \beta_{2}+\alpha_{1} \alpha_{2} \beta_{1}+\alpha_{2}^{2} \delta
\end{array}\right) \\
& D_{2}=\alpha_{1}^{4} \beta_{2}^{2} \eta^{2}+\alpha_{1}^{3} \beta_{2}^{2} \eta^{3}+\alpha_{1}^{4} \alpha_{2}^{3} \eta+\alpha_{1}^{3} \alpha_{2}^{2} \delta^{3}+\alpha_{1}^{2} \alpha_{2}^{2} \delta^{3}+\alpha_{1}^{4} \alpha_{2}^{2} \delta^{2}+2 \alpha_{1}^{3} \alpha_{2}^{3} \delta^{2}+\alpha_{1}^{2} \alpha_{2}^{4} \delta^{2}+\alpha_{1}^{3} \alpha_{2}^{4} \delta+\alpha_{1}^{3} \alpha_{2}^{2} \eta^{3}+\alpha_{1}^{2} \beta_{2}^{3} \eta^{3} \\
& +\alpha_{1}^{2} \alpha_{2}^{3} \eta^{3}+\alpha_{2}^{3} \beta_{1}^{2} \eta^{3}+\alpha_{2}^{2} \beta_{1}^{3} \eta^{3}+2 \alpha_{1}^{3} \alpha_{2} \beta_{2} \eta^{2} \delta+2 \alpha_{1}^{3} \alpha_{2} \beta_{1} \beta_{2} \eta^{2}+4 \alpha_{1}^{2} \alpha_{2}^{2} \beta_{1} \beta_{2} \eta^{2}+\alpha_{1}^{2} \alpha_{2} \beta_{1}^{2} \beta_{2} \eta^{2}+2 \alpha_{1}^{2} \alpha_{2}^{2} \beta_{1}^{2} \eta^{2} \\
& +3 \alpha_{1}^{2} \alpha_{2}^{3} \beta_{1} \eta^{2}+3 \alpha_{1}^{2} \alpha_{2}^{3} \eta^{2} \delta+3 \alpha_{1} \alpha_{2}^{3} \beta_{1}^{2} \eta^{2}+\alpha_{1} \alpha_{2}^{4} \beta_{1} \eta^{2}+\alpha_{2}^{3} \beta_{1}^{2} \eta^{2} \delta+4 \alpha_{1}^{3} \alpha_{2}^{3} \eta \delta+\alpha_{1}^{3} \alpha_{2} \beta_{2}^{3} \eta+2 \alpha_{1}^{4} \alpha_{2}^{2} \eta \delta \\
& +2 \alpha_{1}^{3} \alpha_{2}^{2} \beta_{2}^{2} \eta+3 \alpha_{1}^{2} \alpha_{2}^{3} \eta \delta^{2}+3 \alpha_{1}^{3} \alpha_{2}^{2} \eta \delta^{2}+2 \alpha_{1}^{2} \alpha_{2}^{4} \eta \delta \alpha_{1} \alpha_{2}^{3} \beta_{1}^{3} \eta+\alpha_{1} \alpha_{2}^{4} \beta_{1}^{2} \eta+\alpha_{1}^{4} \alpha_{2} \beta_{2}^{2} \eta+\alpha_{1}^{4} \alpha_{2}^{2} \beta_{2} \eta+2 \alpha_{1}^{3} \alpha_{2}^{3} \beta_{2} \eta \\
& +2 \alpha_{1}^{3} \alpha_{2}^{3} \beta_{1} \eta+2 \alpha_{1}^{2} \alpha_{2}^{3} \beta_{2}^{2} \eta+\alpha_{1}^{2} \alpha_{2}^{4} \beta_{1} \eta 2 \alpha_{1} \alpha_{2}^{3} \beta_{1} \eta^{2} \delta+2 \alpha_{1} \alpha_{2}^{2} \beta_{1}^{2} \eta^{2} \delta+2 \alpha_{1} \alpha_{2}^{3} \beta_{1} \beta_{2} \eta^{2}+\alpha_{1}^{3} \alpha_{2}^{4} \eta+\alpha_{1}^{4} \alpha_{2}^{3} \delta \\
& +\alpha_{1}^{3} \alpha_{2}^{2} \beta_{1} \beta_{2} \delta+\alpha_{1} \alpha_{2}^{3} \beta_{1}^{2} \beta_{2} \eta+2 \alpha_{1}^{3} \alpha_{2}^{2} \beta_{1} \beta_{2} \eta+\alpha_{1}^{2} \alpha_{2}^{2} \beta_{1}^{2} \beta_{2} \eta+4 \alpha_{1}^{2} \alpha_{2}^{2} \beta_{1} \eta^{2} \delta+2 \alpha_{1}^{3} \alpha_{2}^{3} \beta_{1} \delta+\alpha_{1}^{2} \alpha_{2}^{3} \beta_{1}^{2} \delta+\alpha_{1}^{2} \alpha_{2}^{4} \beta_{1} \delta \\
& +\alpha_{1}^{3} \alpha_{2} \beta_{2} \eta^{3}+2 \alpha_{1}^{2} \alpha_{2} \beta_{2}^{2} \eta^{3}+\alpha_{1}^{2} \beta_{1} \beta_{2}^{2} \eta^{3}+2 \alpha_{1}^{2} \alpha_{2}^{2} \beta_{2} \eta^{3}+2 \alpha_{1}^{2} \alpha_{2}^{2} \beta_{1} \eta^{3}+\alpha_{1} \alpha_{2}^{3} \beta_{1} \eta^{3}+\alpha_{1}^{2} \alpha_{2}^{3} \beta_{1} \beta_{2} \delta+2 \alpha_{1}^{2} \alpha_{2} \beta_{1} \beta_{2} \eta^{3} \\
& +\alpha_{1} \alpha_{2} \beta_{1} \beta_{2}^{2} \eta^{3}+\alpha_{1} \alpha_{2} \beta_{1}^{2} \beta_{2} \eta^{3}+2 \alpha_{1} \alpha_{2}^{2} \beta_{1} \beta_{2} \eta^{3}+2 \alpha_{1} \alpha_{2}^{2} \beta_{1}^{2} \eta^{3}+\alpha_{2}^{2} \beta_{1}^{2} \beta_{2} \eta^{3}+\alpha_{2}^{3} \beta_{2}^{2} \beta_{2} \eta^{2}+3 \alpha_{1}^{3} \alpha_{2}^{2} \eta^{2} \delta+\alpha_{1} \alpha_{2}^{2} \beta_{1}^{3} \eta^{2} \\
& +\alpha_{1}^{4} \alpha_{2} \beta_{2} \eta^{2}+\alpha_{1}^{3} \beta_{1} \beta_{2}^{2} \eta^{2}+3 \alpha_{1}^{3} \alpha_{2} \beta_{2}^{2} \eta^{2}+2 \alpha_{1}^{2} \alpha_{2}^{3} \beta_{2} \eta^{2}+\alpha_{1}^{2} \alpha_{2} \beta_{2}^{3} \eta^{2}+\alpha_{1}^{3} \beta_{2}^{2} \eta^{2} \delta+3 \alpha_{1}^{3} \alpha_{2}^{2} \beta_{2} \eta^{2}+2 \alpha_{1}^{3} \alpha_{2}^{2} \beta_{1} \eta^{2} \\
& +2 \alpha_{1}^{2} \alpha_{2}^{2} \beta_{2}^{2} \eta^{2}+2 \alpha_{1}^{2} \alpha_{2} \beta_{2}^{2} \eta^{2} \delta+4 \alpha_{1}^{2} \alpha_{2}^{2} \beta_{2} \eta^{2} \delta+2 \alpha_{1}^{2} \alpha_{2} \beta_{1} \beta_{2} \eta^{2} \delta+2 \alpha_{1} \alpha_{2}^{2} \beta_{1} \beta_{2} \eta^{2} \delta+2 \alpha_{1} \alpha_{2}^{2} \beta_{1}^{2} \beta_{2} \eta^{2}+2 \alpha_{1}^{2} \alpha_{2} \beta_{1} \beta_{2}^{2} \eta^{2} \\
& +\alpha_{1} \alpha_{2}^{2} \beta_{1} \beta_{2}^{2} \eta^{2}+\alpha_{1}^{3} \beta_{2}^{3} \eta^{2}+\alpha_{1}^{4} \alpha_{2}^{2} \eta^{2}+2 \alpha_{1}^{3} \alpha_{2}^{3} \eta^{2}+\alpha_{2}^{3} \beta_{1}^{3} \eta^{2}+\alpha_{2}^{4} \beta_{1}^{2} \eta^{2}+\alpha_{1}^{2} \alpha_{2}^{4} \eta^{2}+2 \alpha_{1}^{3} \alpha_{2}^{3} \beta_{1} \delta+\alpha_{1}^{2} \alpha_{2}^{3} \beta_{1}^{2} \delta \\
& +\alpha_{1}^{2} \alpha_{2}^{4} \beta_{1} \delta+\alpha_{1}^{3} \alpha_{2} \beta_{2} \eta^{3}+2 \alpha_{1}^{2} \alpha_{2} \beta_{2}^{2} \eta^{3}+2 \alpha_{1}^{2} \alpha_{2}^{2} \beta_{2} \eta^{3}+2 \alpha_{1}^{2} \alpha_{2}^{2} \beta_{1} \eta^{3}+\alpha_{1} \alpha_{2}^{3} \beta_{1} \eta^{3}+\alpha_{1}^{2} \alpha_{2}^{3} \beta_{1} \beta_{2} \delta+2 \alpha_{1}^{2} \alpha_{2} \beta_{1} \beta_{2} \eta^{3} \\
& +\alpha_{1} \alpha_{2} \beta_{1} \beta_{2}^{2} \eta^{3}+\alpha_{1} \alpha_{2} \beta_{1}^{2} \beta_{2} \eta^{3}+2 \alpha_{1} \alpha_{2}^{2} \beta_{1} \beta_{2} \eta^{3}+2 \alpha_{1} \alpha_{2}^{2} \beta_{1}^{2} \eta^{3}+\alpha_{2}^{2} \beta_{1}^{2} \beta_{2} \eta^{3}+\alpha_{2}^{3} \beta_{2}^{2} \beta_{2} \eta^{2}+3 \alpha_{1}^{3} \alpha_{2}^{2} \eta^{2} \delta \\
& +\alpha_{1} \alpha_{2}^{2} \beta_{1}^{3} \eta^{2}+\alpha_{1}^{4} \alpha_{2} \beta_{2} \eta^{2}+\alpha_{1}^{3} \beta_{1} \beta_{2}^{2} \eta^{2}+3 \alpha_{1}^{3} \alpha_{2} \beta_{2}^{2} \eta^{2}+2 \alpha_{1}^{2} \alpha_{2}^{3} \beta_{2} \eta^{2}+\alpha_{1}^{2} \alpha_{2} \beta_{2}^{3} \eta^{2}+\alpha_{1}^{3} \beta_{2}^{2} \eta^{2} \delta+3 \alpha_{1}^{3} \alpha_{2}^{2} \beta_{2} \eta^{2} \\
& +2 \alpha_{1}^{3} \alpha_{2}^{2} \beta_{1} \eta^{2}+2 \alpha_{1}^{2} \alpha_{2}^{2} \beta_{2}^{2} \eta^{2}+2 \alpha_{1}^{2} \alpha_{2} \beta_{2}^{2} \eta^{2} \delta+4 \alpha_{1}^{2} \alpha_{2}^{2} \beta_{2} \eta^{2} \delta+2 \alpha_{1}^{2} \alpha_{2} \beta_{1} \beta_{2} \eta^{2} \delta+2 \alpha_{1} \alpha_{2}^{2} \beta_{1} \beta_{2} \eta^{2} \delta+2 \alpha_{1} \alpha_{2}^{2} \beta_{1}^{2} \beta_{2} \eta^{2} \\
& +2 \alpha_{1}^{2} \alpha_{2} \beta_{1} \beta_{2}^{2} \eta^{2}+\alpha_{1} \alpha_{2}^{2} \beta_{1} \beta_{2}^{2} \eta^{2}+\alpha_{1}^{3} \beta_{2}^{3} \eta^{2}+\alpha_{1}^{4} \alpha_{2}^{2} \eta^{2}+2 \alpha_{1}^{3} \alpha_{2}^{3} \eta^{2}+\alpha_{2}^{3} \beta_{1}^{3} \eta^{2}+\alpha_{2}^{4} \beta_{1}^{2} \eta^{2}+\alpha_{1}^{2} \alpha_{2}^{4} \eta^{2}+\alpha_{1}^{2} \beta_{1} \beta_{2}^{2} \eta^{3}
\end{aligned}
$$

\subsection{Busy Period Analysis}

Using the initial condition in subsection 4.1 above as for reliability case and (5) and (6), the steady-state busy period is

$$
B(\infty)=1-P_{0}(\infty)=\frac{N_{3}}{D_{2}}
$$


where

\subsection{Profit Analysis}

The system/units are subjected to corrective maintenance at failure as can be observed in states 1-8. From Figure 1, the repairman is busy performing corrective maintenance action to the units/system at failure in states 1-8. According to $[8,9]$, the expected profit per unit time incurred to the system in the steady-state is given by: Profit $=$ total revenue generated - cost incurred for repairing the failed units.

$$
P F=C_{0} A(\infty)-C_{1} B(\infty)
$$

where $\mathrm{PF}_{2}$ : is the profit incurred to the system, $\mathrm{C}_{0}$ : is the revenue per unit up time of the system, $C_{1}$ : is the cost per unit time which the system is under repair.

\section{Results and Discussions}

In this section, we numerically obtained the results for mean time to system failure, system availability, busy period and profit function for all the developed models. For the model analysis, the following set of parameters values are fixed throughout the simulations for consistency: $\beta_{1}=0.05, \beta_{2}=0.2, \alpha_{1}=0.5, \alpha_{2}=0.01$, $\delta=0.1, \eta=0.5$ in Figures 3-7 and assumed $\alpha_{2}=$ 0.7, in Figures 8-17, $C_{0}=900,000, C_{1}=100,000$ in Figures 14-17.

Effect of $\delta$ on MTSF, steady-state availability, busy period and profit can be observed in Figures 5, 6, 11 and 16. From Figures 5, $\mathbf{6}$ and 16, it is evident that the MTSF, availability and profit decrease as $\delta$ increases while from Figure 11 the busy period increases as $\delta$ increase. Similar results can be observed in Figures 4, 7, 13 and 15 with respect to $\beta_{1}$.

In these figures, MTSF, availability and profit decrease as $\beta_{1}$ increases while busy period increase with

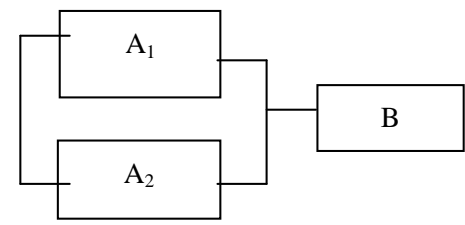

Figure 2. Reliability block diagram of the system.

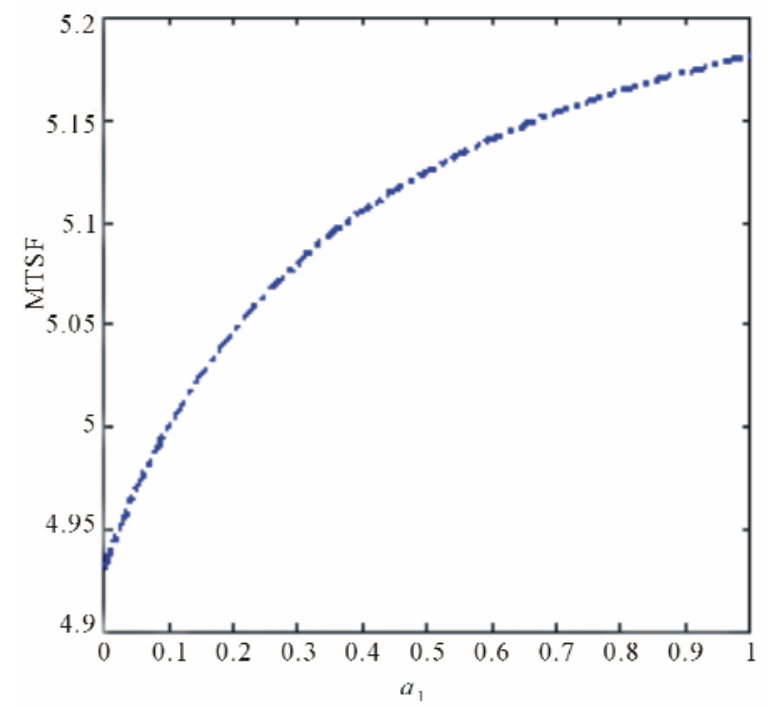

Figure 3. Effect of $\alpha_{1}$ on MTSF.

$$
\begin{aligned}
& N_{3}=\alpha_{1}^{4} \beta_{2}^{2} \eta^{2}+\alpha_{1}^{3} \beta_{2}^{2} \eta^{3}+\alpha_{1}^{4} \alpha_{2}^{3} \eta+\alpha_{1}^{3} \alpha_{2}^{2} \delta^{3}+\alpha_{1}^{2} \alpha_{2}^{2} \delta^{3}+\alpha_{1}^{4} \alpha_{2}^{2} \delta^{2}+2 \alpha_{1}^{3} \alpha_{2}^{3} \delta^{2}+\alpha_{1}^{2} \alpha_{2}^{4} \delta^{2}+\alpha_{1}^{3} \alpha_{2}^{4} \delta+\alpha_{1}^{3} \alpha_{2}^{2} \eta^{3} \\
& +\alpha_{1}^{2} \beta_{2}^{3} \eta^{3}+\alpha_{1}^{2} \alpha_{2}^{3} \eta^{3}+\alpha_{2}^{3} \beta_{1}^{2} \eta^{3}+\alpha_{2}^{2} \beta_{1}^{3} \eta^{3}+2 \alpha_{1}^{3} \alpha_{2} \beta_{2} \eta^{2} \delta+2 \alpha_{1}^{3} \alpha_{2} \beta_{1} \beta_{2} \eta^{2}+4 \alpha_{1}^{2} \alpha_{2}^{2} \beta_{1} \beta_{2} \eta^{2}+\alpha_{1}^{2} \alpha_{2} \beta_{1}^{2} \beta_{2} \eta^{2} \\
& +2 \alpha_{1}^{2} \alpha_{2}^{2} \beta_{1}^{2} \eta^{2}+3 \alpha_{1}^{2} \alpha_{2}^{3} \beta_{1} \eta^{2}+3 \alpha_{1}^{2} \alpha_{2}^{3} \eta^{2} \delta+3 \alpha_{1} \alpha_{2}^{3} \beta_{1}^{2} \eta^{2}+\alpha_{1} \alpha_{2}^{4} \beta_{1} \eta^{2}+\alpha_{2}^{3} \beta_{1}^{2} \eta^{2} \delta+4 \alpha_{1}^{3} \alpha_{2}^{3} \eta \delta+\alpha_{1}^{3} \alpha_{2} \beta_{2}^{3} \eta \\
& +2 \alpha_{1}^{4} \alpha_{2}^{2} \eta \delta+2 \alpha_{1}^{3} \alpha_{2}^{2} \beta_{2}^{2} \eta+3 \alpha_{1}^{2} \alpha_{2}^{3} \eta \delta^{2}+3 \alpha_{1}^{3} \alpha_{2}^{2} \eta \delta^{2}+2 \alpha_{1}^{2} \alpha_{2}^{4} \eta \delta+\alpha_{1} \alpha_{2}^{3} \beta_{1}^{3} \eta+\alpha_{1} \alpha_{2}^{4} \beta_{1}^{2} \eta+\alpha_{1}^{4} \alpha_{2} \beta_{2}^{2} \eta \\
& +\alpha_{1}^{4} \alpha_{2}^{2} \beta_{2} \eta+2 \alpha_{1}^{3} \alpha_{2}^{3} \beta_{2} \eta+2 \alpha_{1}^{3} \alpha_{2}^{3} \beta_{1} \eta+2 \alpha_{1}^{2} \alpha_{2}^{3} \beta_{2}^{2} \eta+\alpha_{1}^{2} \alpha_{2}^{4} \beta_{1} \eta+4 \alpha_{1}^{2} \alpha_{2}^{2} \beta_{1} \eta^{2} \delta+2 \alpha_{1} \alpha_{2}^{3} \beta_{1} \eta^{2} \delta+2 \alpha_{1} \alpha_{2}^{2} \beta_{1}^{2} \eta^{2} \delta \\
& +2 \alpha_{1} \alpha_{2}^{3} \beta_{1} \beta_{2} \eta^{2}+\alpha_{1}^{3} \alpha_{2}^{4} \eta+\alpha_{1}^{4} \alpha_{2}^{3} \delta+\alpha_{1}^{3} \alpha_{2}^{2} \beta_{1} \beta_{2} \delta+\alpha_{1} \alpha_{2}^{3} \beta_{1}^{2} \beta_{2} \eta+2 \alpha_{1}^{3} \alpha_{2}^{2} \beta_{1} \beta_{2} \eta+\alpha_{1}^{2} \alpha_{2}^{2} \beta_{1}^{2} \beta_{2} \eta+\alpha_{1}^{3} \alpha_{2} \beta_{2} \eta \delta^{2} \\
& +2 \alpha_{1}^{2} \alpha_{2}^{2} \beta_{2} \eta \delta^{2}+2 \alpha_{1}^{2} \alpha_{2}^{2} \beta_{1} \eta \delta^{2}+\alpha_{1}^{2} \alpha_{2}^{2} \beta_{1} \beta_{2}^{2} \eta+\alpha_{1}^{4} \alpha_{2} \beta_{2} \eta \delta+5 \alpha_{1}^{3} \alpha_{2}^{2} \beta_{2} \eta \delta+3 \alpha_{1}^{3} \alpha_{2}^{2} \beta_{1} \eta \delta+2 \alpha_{1}^{2} \alpha_{2}^{2} \beta_{2}^{2} \eta \delta \\
& +2 \alpha_{1}^{2} \alpha_{2}^{2} \beta_{1}^{2} \eta \delta+2 \alpha_{1}^{3} \alpha_{2} \beta_{2}^{2} \eta \delta+\alpha_{1}^{3} \alpha_{2} \beta_{1} \beta_{2} \eta \delta+4 \alpha_{1}^{2} \alpha_{2}^{2} \beta_{1} \beta_{2} \eta \delta+5 \alpha_{1}^{2} \alpha_{2}^{3} \beta_{1} \eta \delta+\alpha_{1} \alpha_{2}^{3} \beta_{1} \eta \delta^{2}+\alpha_{1} \alpha_{2}^{4} \beta_{1} \eta \delta \\
& +2 \alpha_{1} \alpha_{2}^{3} \beta_{1}^{2} \eta \delta+\alpha_{1}^{3} \alpha_{2} \beta_{1} \beta_{2}^{2} \eta+\alpha_{1} \alpha_{2}^{3} \beta_{1} \beta_{2} \eta \delta+3 \alpha_{1}^{2} \alpha_{2}^{3} \beta_{2} \eta \delta+2 \alpha_{1}^{2} \alpha_{2}^{3} \beta_{1} \beta_{2} \eta+2 \alpha_{1}^{3} \alpha_{2}^{2} \beta_{2} \delta^{2}+\alpha_{1}^{3} \alpha_{2}^{2} \beta_{1} \delta^{2} \\
& +\alpha_{1}^{2} \alpha_{2}^{3} \beta_{2} \delta^{2}+2 \alpha_{1}^{2} \alpha_{2}^{3} \beta_{1} \delta^{2}+\alpha_{1}^{4} \alpha_{2}^{2} \beta_{2} \delta+\alpha_{1}^{3} \alpha_{2}^{2} \beta_{2}^{2} \delta+2 \alpha_{1}^{3} \alpha_{2}^{3} \beta_{2} \delta+2 \alpha_{1}^{3} \alpha_{2}^{3} \beta_{1} \delta+\alpha_{1}^{2} \alpha_{2}^{3} \beta_{1}^{2} \delta+\alpha_{1}^{2} \alpha_{2}^{4} \beta_{1} \delta \\
& +\alpha_{1}^{3} \alpha_{2} \beta_{2} \eta^{3}+2 \alpha_{1}^{2} \alpha_{2} \beta_{2}^{2} \eta^{3}+\alpha_{1}^{2} \beta_{1} \beta_{2}^{2} \eta^{3}+2 \alpha_{1}^{2} \alpha_{2}^{2} \beta_{2} \eta^{3}+2 \alpha_{1}^{2} \alpha_{2}^{2} \beta_{1} \eta^{3}+\alpha_{1} \alpha_{2}^{3} \beta_{1} \eta^{3}+\alpha_{1}^{2} \alpha_{2}^{3} \beta_{1} \beta_{2} \delta+2 \alpha_{1}^{2} \alpha_{2} \beta_{1} \beta_{2} \eta^{3} \beta_{1}^{2} \eta^{3} \\
& +\alpha_{1} \alpha_{2} \beta_{1} \beta_{2}^{2} \eta^{3}+\alpha_{1} \alpha_{2} \beta_{1}^{2} \beta_{2} \eta^{3}+2 \alpha_{1} \alpha_{2}^{2} \beta_{1} \beta_{2} \eta^{3}+2 \alpha_{1} \alpha_{2}^{2}+\alpha_{2}^{2} \beta_{1}^{2} \beta_{2} \eta^{3}+\alpha_{2}^{3} \beta_{2}^{2} \beta_{2} \eta^{2}+3 \alpha_{1}^{3} \alpha_{2}^{2} \eta^{2} \delta+\alpha_{1} \alpha_{2}^{2} \beta_{1}^{3} \eta^{2} \\
& +\alpha_{1}^{4} \alpha_{2} \beta_{2} \eta^{2}+\alpha_{1}^{3} \beta_{1} \beta_{2}^{2} \eta^{2}+3 \alpha_{1}^{3} \alpha_{2} \beta_{2}^{2} \eta^{2}+2 \alpha_{1}^{2} \alpha_{2}^{3} \beta_{2} \eta^{2}+\alpha_{1}^{2} \alpha_{2} \beta_{2}^{3} \eta^{2}+\alpha_{1}^{3} \beta_{2}^{2} \eta^{2} \delta+3 \alpha_{1}^{3} \alpha_{2}^{2} \beta_{2} \eta^{2}+2 \alpha_{1}^{3} \alpha_{2}^{2} \beta_{1} \eta^{2} \\
& +2 \alpha_{1}^{2} \alpha_{2}^{2} \beta_{2}^{2} \eta^{2}+2 \alpha_{1}^{2} \alpha_{2} \beta_{2}^{2} \eta^{2} \delta+4 \alpha_{1}^{2} \alpha_{2}^{2} \beta_{2} \eta^{2} \delta+2 \alpha_{1}^{2} \alpha_{2} \beta_{1} \beta_{2} \eta^{2} \delta+2 \alpha_{1} \alpha_{2}^{2} \beta_{1} \beta_{2} \eta^{2} \delta+2 \alpha_{1} \alpha_{2}^{2} \beta_{1}^{2} \beta_{2} \eta^{2}+2 \alpha_{1}^{2} \alpha_{2} \beta_{1} \beta_{2}^{2} \eta^{2} \\
& +\alpha_{1} \alpha_{2}^{2} \beta_{1} \beta_{2}^{2} \eta^{2}+\alpha_{1}^{3} \beta_{2}^{3} \eta^{2}+\alpha_{1}^{4} \alpha_{2}^{2} \eta^{2}+2 \alpha_{1}^{3} \alpha_{2}^{3} \eta^{2}+\alpha_{2}^{3} \beta_{1}^{3} \eta^{2}+\alpha_{2}^{4} \beta_{1}^{2} \eta^{2}+\alpha_{1}^{2} \alpha_{2}^{4} \eta^{2}
\end{aligned}
$$




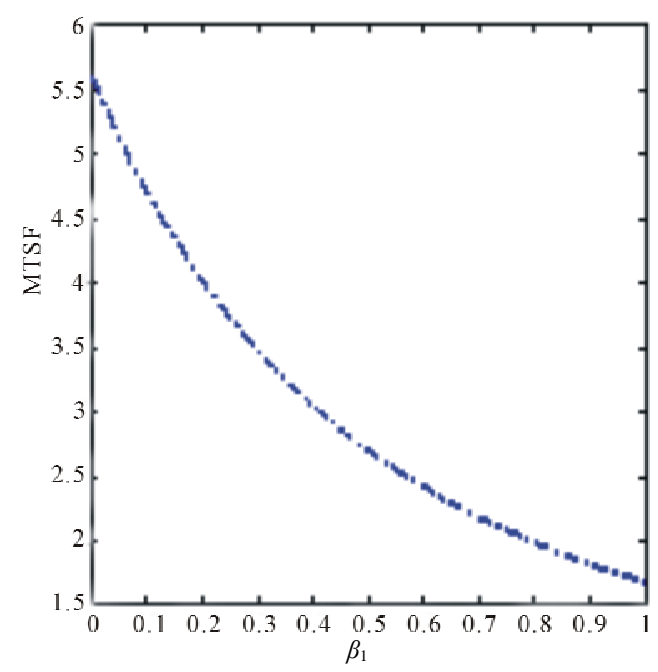

Figure 4. Effect of $\beta_{1}$ on MTSF

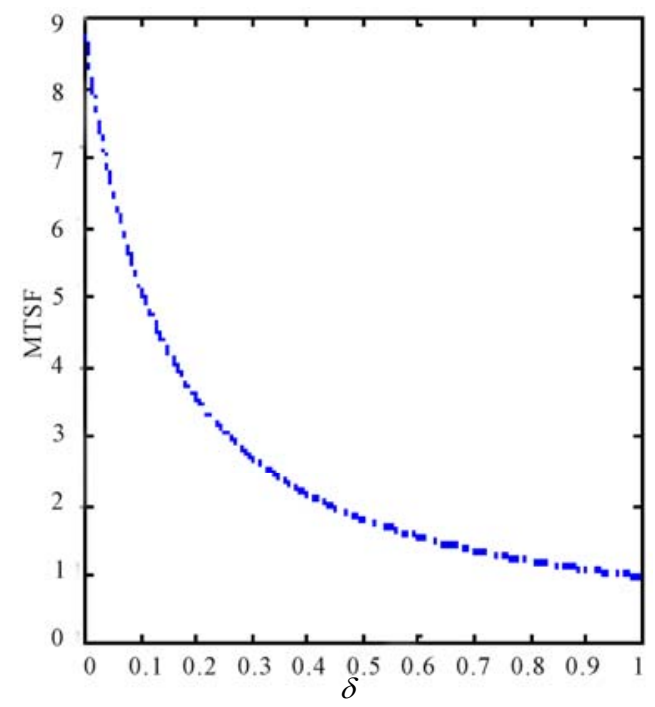

Figure 5. Effect of $\delta$ on MTSF.

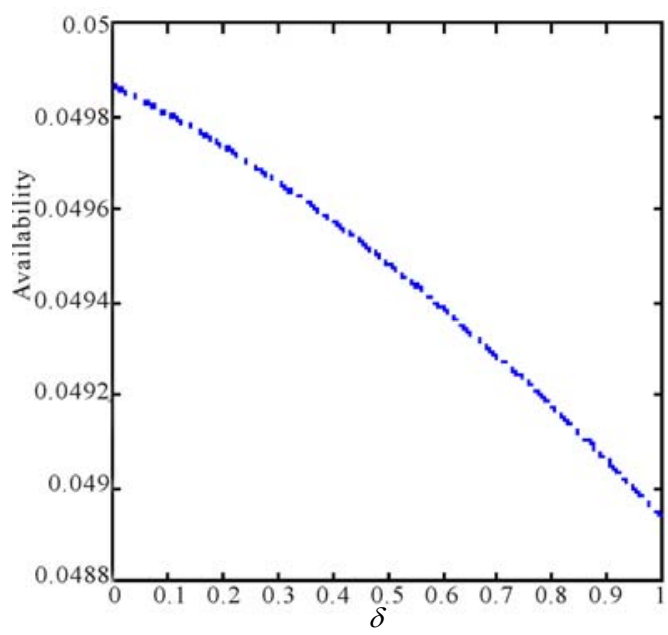

Figure 6. Effect of $\delta$ on availability.

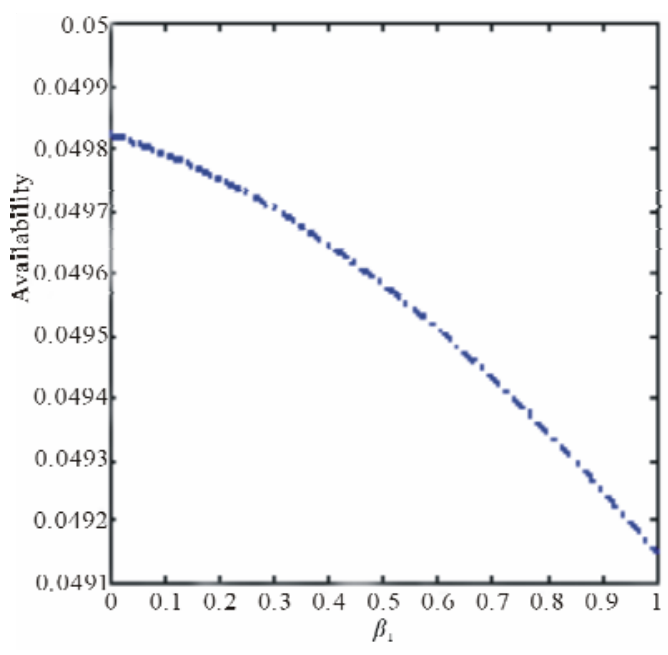

Figure 7. effect of $\beta_{1}$ on availability.

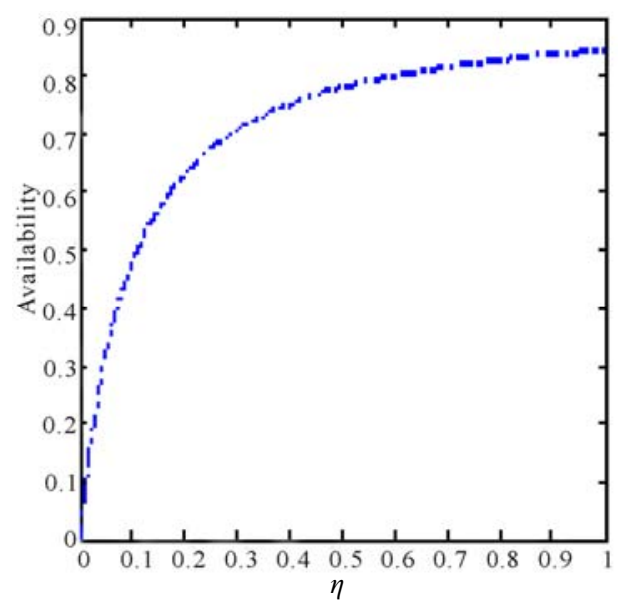

Figure 8. Effect of $\eta$ on availability.

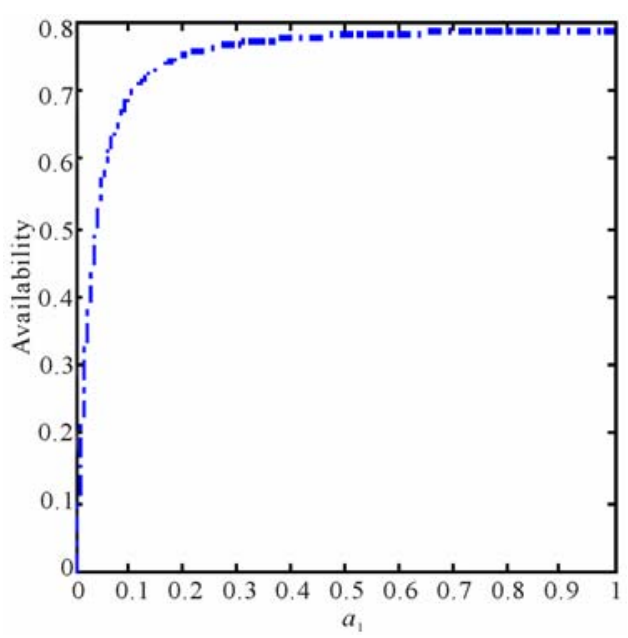

Figure 9. Effect of $\alpha_{1}$ on availability.

increase in $\beta_{1}$ as can be seen in Figure 13. Results of MTSF, steady-state availability, busy period and profit 


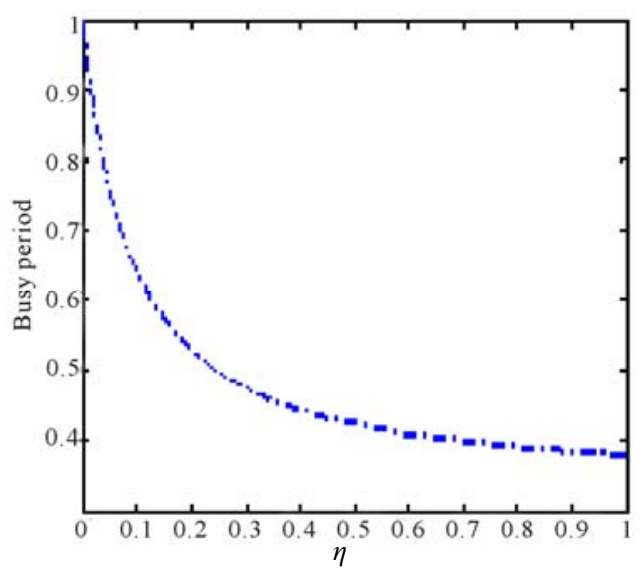

Figure 10. Effect of $\eta$ on busy period.

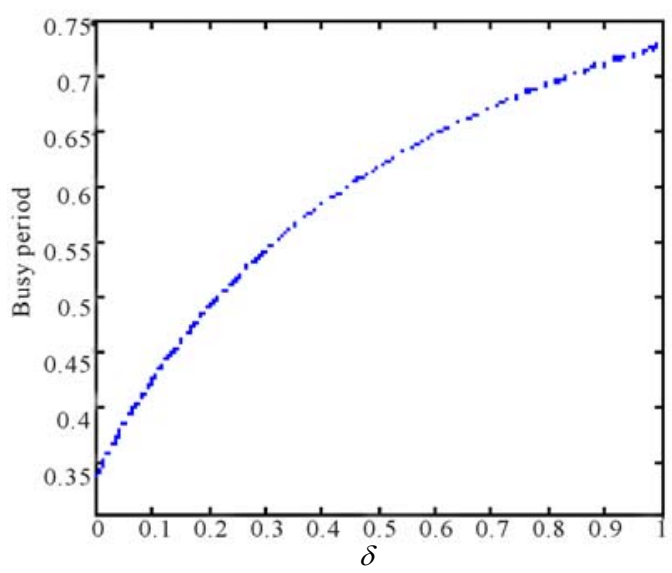

Figure 11. Effect of $\delta$ on busy period.

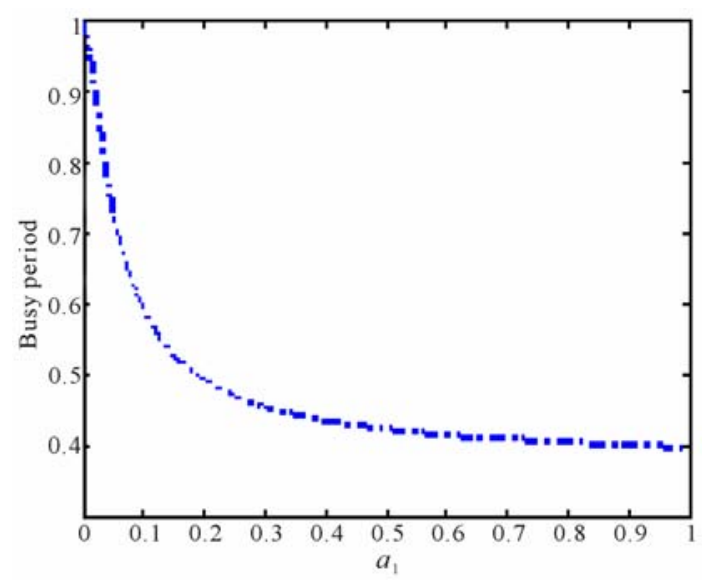

Figure 12. Effect of $\alpha_{1}$ on busy period.

with respect to $\alpha_{1}$ are given in Figures 3, 9, 12 and 14. It is evident from Figures 3, 9 and $\mathbf{1 4}$ that as $\alpha_{1}$ increases, the MTSF, availability and profit also increases while busy period decreases as $\alpha_{1}$ increases in Figure 12. Furthermore, the impact of $\eta$ on availability, busy period and profit can be seen in Figures 8, 10 and 17. From
Figures 8 and 17, availability and profit increase as $\eta$ increases and from Figure $\mathbf{1 0}$ the busy period decreases as $\eta$ increases.

\section{Conclusion}

In this paper, we constructed a repairable system with two subsystems A and B in series. Subsystem A has two units $A_{1}$ and $A_{2}$ in active parallel while subsystem $B$ is a single unit. We have developed the explicit expressions for the MTSF, availability, busy period and profit function. We performed a parametric investigation of various system parameters on MTSF, system availability and profit function and captured their effects on MTSF, availability, busy period and profit function. This is the main contribution of the paper.

\section{REFERENCES}

[1] K. M. El-Said and M. S. EL-Sherbeny, "Evaluation of Reliability and Availability Characteristics of Two Different Systems by Using Linear First Order Differential Equations,” Journal of Mathematics and Statistics, Vol. 1, No. 2, 2005, pp. 119-123. http://dx.doi.org/10.3844/jmssp.2005.119.123

[2] I. Yusuf and N. Hussaini, "Evaluation of Reliability and Availability Characteristics of 2-Out of -3 Standby System under a Perfect Repair Condition,” American Journal of Mathematics and Statistics, Vol. 2, No. 5, 2012, pp. 114-119. http://dx.doi.org/10.5923/j.ajms.20120205.03

[3] S. V. Amari, J. B. Dugan and R. B. Misra, "Optimal Reliability of Systems Subject to Imperfect Fault-Coverage," IEEE Transactions on Reliability, Vol. 48, No. 3, 1999, pp. 275-284. http://dx.doi.org/10.1109/24.799899

[4] K.-H. Wang and B. D. Sivazlian, "Reliability of System with Warm Standbys and Repairmen," Microelectronics and Reliability, Vol. 29, No. 5, 1989, pp. 849-860. http://dx.doi.org/10.1016/0026-2714(89)90184-4

[5] J.-C. Ke and K.-H. Wang, "The Reliability Analysis of Balking and Reneging in a Repairable System with Warm Standbys," Quality and Reliability Engineering International, Vol. 18, No. 6, 2002, pp. 467-478. http://dx.doi.org/10.1002/qre.495

[6] K.-H. Wang and J.-C. Ke, "Probabilistic Analysis of a Repairable System with Warm Standbys Plus Balking and Reneging," Applied Mathematical Modeling, Vol. 27, No. 4, 2003, pp. 327-336. http://dx.doi.org/10.1016/S0307-904X(02)00133-6

[7] K.-H. Wang, Y.-J. Lai and J.-B. Ke, "Reliability and Sensitivity Analysis of a System with Warm Standbys and a Repairable Service Station," International Journal of Operations Research, Vol. 1, No.1, 2004, pp. 61-70

[8] K. M. El-Said, "Cost Analysis of a System with Preventive Maintenance by Using Kolmogorov's forward Equations Method". American Journal of Applied Sciences, Vol.5, No.4, 2008, 405-410.

[9] M. Y. Haggag. "Cost Analysis of a System Involving 
Common Cause Failures and Preventive Maintenance”. Journal of Mathematics and Statistics, Vol. 5, No.4, 2009. pp 305-310.

http://dx.doi.org/10.3844/jmssp.2009.305.310

[10] M. Y. Haggag, “Cost Analysis of k-Out-of-n Repairable
System with Dependent Failure and Standby Support Using Kolmogorov's Forward Equations Method," Journal of Mathematics and Statistics, Vol. 5, No. 4, 2009. pp. 401-407. 